\title{
Influences on Exercise and Sports Participation in College-Aged Students
}

Kelley Henry, Charlotte F. Sanborn, FACSM, Terry Senne, and David L. Nichols, FACSM

Texas Woman's University, Denton, TX

Classification: Doctoral

PURPOSE: The purpose of the study was to determine the reasons why female college students decide to be physically active or not and whether the female student population is familiar with the Female Athlete Triad (TRIAD). The TRIAD is defined by the following conditions: low energy availability, amenorrhea, and osteoporosis. METHODS: Participants were recruited from a university in North Texas to complete a 16-item online survey. Participants were asked to identify the influences and reasons why they did or did not lead a physically active lifestyle. Participants were also questioned about their knowledge of the TRIAD. Data were analyzed via frequency distributions using SPSS version 15.0. RESULTS: Participants included 83 collegiate students but only 63 participants ( 3 males, 60 females) aged $18-49$ (mean $\pm S D=24$ \pm 6.2 years) had complete data that were analyzed. The data of the male participants were not included in the frequency distributions. The three male participants did not have knowledge of the TRIAD. A total of 43 female participants responded when asked if they knew of the TRIAD. Only $18(29 \%)$ of the 63 respondents had knowledge of the TRIAD, nine of whom identified the components of the TRIAD correctly. Six of the nine respondents were Kinesiology majors and two were athletes. The prime influence for participating in exercise, sports or physical activity was self-motivation (30\%). The main reasons students chose to be physically active were for psychological improvement $(30 \%)$, physical appearance (29\%), and enjoyment (26\%). Students chose not to participate in physical activity primarily for the following reasons: low motivation (46\%) and time constraints (35\%). Only two participants selected a potential concern that exercise would have a negative impact on their health. CONCLUSION: Students choose to be physically active based on self-motivation and were not deterred from exercise by issues related to the Female Athlete Triad. 